\title{
Experimental validation of a new magnetic method applied to diagnose a low voltage breaker arc
}

\author{
N. Machkour ${ }^{1}$, Elm. Kheddioui ${ }^{2}$, and A. Bourjillate ${ }^{2}$ \\ ${ }^{1}$ Ecole National des Arts et Métiers de Casablanca, Université Hassan II - B.P. 150 - Mohammedia, Maroc. \\ ${ }^{2}$ Laboratoire d'Optoélectronique, Analyse Optique et Simulation, Université Hassan II - B.P. 150 - Mohammedia, Maroc.
}

\begin{abstract}
In this paper, we present an original method, which allows reconstructing the current density in a low voltage breaker arc. This method is based on the deconvolution of magnetic induction measurements and a function depending on the experimental device geometry. For this study, we propose to use an iterative deconvolution method using minimisation by gradient.
\end{abstract}

\section{Introduction}

Manufacturers of circuit breakers need to know the arc's size, current density, and whether it is diffuse or concentrated, because the mode of the arc (diffuse or concentrated) influences the erosion of the contacts [1-2]. The size of arc can be characterized by optical or magnetic methods. The optical methods give an image of the arc size [3] but their use requires the introduction of transparent walls, which can influence the physicalchemical properties of the arc. Bouvier [4] proposed a magnetic method to determine the current density in plasma. Although this method is not disruptive, it presents the inconvenience to suppose that the arc moves with a constant speed; that is not always realistic.

In this paper, we propose a non-intrusive magnetic method based on deconvolution of magnetic signals and a function depending on the experimental device geometry. At a given moment, it reconstructs the current density, going into a low voltage breaker arc, allowing us to determine the arc size, to characterize its mode and to study its dynamic. A validation of the method is experimentally obtained using the reconstruction of the current density flowing in a stationary conductor.

\section{Modeling and formulation of the problem}

\subsection{Experimental Device}

The experimental device used in this study is already presented in [5-6]. It is constituted of (figure 1):

- breaking device,

- power source,

- 64 Hall Effect sensors placed on both sides of the moving electric arc. These are used to measure magnetic induction along the $\mathrm{Oz}$ direction,

- four multiplexing cards which allow conditioning and switching of measurements,

- Acquisition system to store and treat measurements.

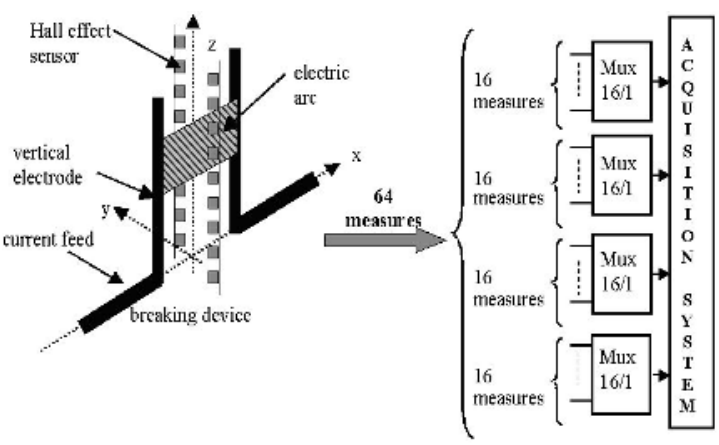

Fig.1. A schematic of the experimental device.

\subsection{Principle of the method}

The method consists of reconstructing the current density distribution along the $\mathrm{Oz}$ direction in low voltage breaker arc. For this purpose, we use a set of vertical measurements of the magnetic induction, which occur on both sides of the electric arc. In order to simplify the mathematical form, the column of the arc is modeled as a rectangular current sheet, composed of a mix of threadlike currents segments; the other elements of the breaking device are compared to rectilinear threadlike segment. This assumption is justified by the fact that the magnetic sensors are placed at a quite distance from the breaking device, and it was experimentally confirmed in earlier work $[3,7]$. 
Initially we suppose that the electric arc is a rectilinear current segment placed in the $\mathrm{xOz}$ plane at the mark $\mathrm{Z}$ (figure 2). Crossed by a current I, this circuit creates a magnetic induction at a point $\mathrm{S}(0, \mathrm{ys}, \mathrm{z})$, located at a distance $y s$ in the $x O z$ plane. See figure 3. The component bzt of this induction in the $\mathrm{Oz}$ direction results from passage of the current $I$ in a circuit consisting of the two current feeds, noted "circuit a", and the electric arc of variable geometry noted "circuit b". The vertical electrodes do not have a magnetic contribution along the $\mathrm{Oz}$ direction.

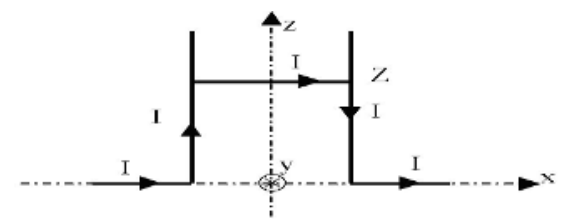

Fig. 2. A simplified electrical circuit for the breaking device.

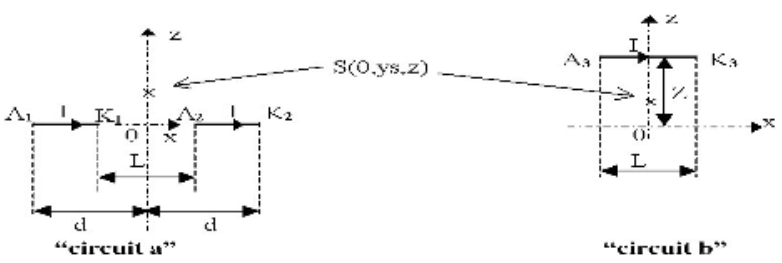

Fig.3. An equivalent diagram of the figure 2 circuit. Thus $b_{z t}(z)=b_{z a}(z)+b_{z b}(z)$ where $b_{z a}(z)$ is the induction created by "circuit a" along Oz direction and $b_{z b}(z)$ is the induction created by "circuit $b$ " along the $\mathrm{Oz}$ direction.

These two inductions are calculated from Biot-Savart's law, for the calculation details see [5-6], $b_{z b}$ depends only on the relative position $\mathrm{z}-\mathrm{Z}$ of the arc with the respect to the measurement point $\mathrm{S}$; and $b_{z a}$ depends only on the position of the point $S$.

$b_{z d}(z)=\frac{\mu_{0} \cdot I \cdot y s}{4 \cdot \pi \cdot\left(y s^{2}+z^{2}\right)} \cdot\left[\frac{2 \cdot d}{\sqrt{y s^{2}+(d)^{2}+z^{2}}}+\frac{-L}{\sqrt{y s^{2}+\left(\frac{L}{2}\right)^{2}+z^{2}}}\right]$

$=f(z) I$,

$b_{z b}(z)=\frac{\mu_{0} \cdot I \cdot y s}{4 \cdot \pi \cdot\left(y s^{2}+(z-Z)^{2}\right)} \cdot\left[\frac{L}{\sqrt{y s^{2}+\left(\frac{L}{2}\right)^{2}+(z-Z)^{2}}}\right]=g(z-Z) \cdot I$,

Now, let us consider that the arc is composed of $\mathrm{N}$ parallel and rectilinear segments. Each segment placed at mark $Z_{j}$ is crossed by a current $i\left(Z_{j}\right)$. In this case $b_{z t}$ is equal to $b_{z t}(z)=f(z) \cdot I+\sum_{j=1}^{N} g\left(z-Z_{j}\right) \cdot i\left(Z_{j}\right)$.

$\mathrm{b}_{\mathrm{zb}}(\mathrm{z})=\mathrm{b}_{\mathrm{zt}}(\mathrm{z})-\mathrm{f}(\mathrm{z}) \cdot \mathrm{I}=\sum_{\mathrm{j}=1}^{\mathrm{N}} \mathrm{g}\left(\mathrm{z}-\mathrm{Z}_{\mathrm{j}}\right) \cdot \mathrm{i}\left(\mathrm{Z}_{\mathrm{j}}\right)=g(z) * i(z)$
So the induction created by the electric arc is a convolution product of the function $g(z)$ by the current $i(z)$. The function $g(z)$ represents the magnetic induction of circuit 2 when the current segment is at $\mathrm{Z}=0$ and crossed by a current of $1 \mathrm{~A}$. Using the usual deconvolution methods, the current density i(z) may be found.

Magnetic induction is measured in the $\mathrm{Oz}$ direction using a step of $1.5 \mathrm{~mm}$. In Equation (4) the variable $\mathrm{z}$ can be replaced by the variable $\mathrm{k}$, with $\mathrm{k}=\mathrm{z} / 1.5$ and $\mathrm{K}=\mathrm{Z} / 1.5$. The following relation is obtained:

$b_{z b}(k)=b_{z t_{\text {mes }}}(k)-f(k) \cdot I=\sum_{K=1}^{N} g(k-K) \cdot i(K)$.

where $b_{z b}(k)$ is the magnetic induction created by the arc, $b_{z t}(k)$ is the total magnetic induction created by the breaker device and measured by the sensor which is placed at $\mathrm{z}=\mathrm{kx} 1.5 \mathrm{~mm}$, and $\mathrm{I}$ is the total current. The relation (5) represents the total magnetic induction created by $\mathrm{N}$ current segments at one point of the $\mathrm{Oz}$ axis. For $\mathrm{N}$ points the following matrix is obtained:

$\left[\begin{array}{c}\mathrm{b}_{\mathrm{zb}}(0) \\ \vdots \\ \mathrm{b}_{\mathrm{zb}}(\mathrm{N}-1)\end{array}\right]=\left[\begin{array}{ccc}\mathrm{g}(0) & \cdots & \mathrm{g}(\mathrm{N}-1) \\ \vdots & \ddots & \vdots \\ \mathrm{g}(\mathrm{N}-1) & \cdots & \mathrm{g}(0)\end{array}\right]\left[\begin{array}{c}\mathrm{i}(0) \\ \vdots \\ \mathrm{i}(\mathrm{N}-1)\end{array}\right]$,

That is to say: $\quad \mathrm{B}_{\mathrm{zb}}=\mathbf{G} \times \mathbf{I}$.

where $\boldsymbol{B}_{z b}$ is the magnetic induction vector, and $\mathbf{I}$ is the current density vector, which is to be determined. The calculation of the current density $\mathrm{i}(\mathrm{k})$ consists of solving the system (6). The matrix $\mathbf{G}$ is a Toeplitz's matrix, and all of its elements are located on parallels at the diagonal and all are identical. Moreover $\mathbf{G}$ is a symmetrical and square matrix. The conditioning factor of this matrix, defined by: $\operatorname{Cond}(\mathbf{G})=\|\mathbf{G}\| x\left\|\mathbf{G}^{-\mathbf{1}}\right\|$ is much higher than 1. Thus the matrix $\mathbf{G}$ is ill-conditioned. Due to the noisy data, a direct calculation of the matrix inverse $\mathbf{G}^{-\mathbf{1}}$ will lead to problems linked to the instability of the solutions. To solve this system we need to use optimization methods, such as iterative method based on the minimization by gradient [8-9].

The principle of this method starts with an estimate $\mathbf{I}_{\mathbf{0}}$ of the vector $\mathbf{I}$ to determine the successive vectors $\mathbf{I}_{1}, \mathbf{I}_{2}$ etc. The starting vector $\mathbf{I}_{\mathbf{0}}$ presents a uniform distribution of the current divided into $\mathrm{N}$ elements and covers the interelectrode space. Each element represents a current segment crossed by a current equal to $\frac{\mathrm{I}}{\mathrm{N}}$, where $I$ is the total current. The solution of the system requires that the criterion [8]:

$$
J(\mathbf{I})=\frac{1}{2} \cdot\left[\left(\mathbf{B}_{\mathbf{z b}}-\mathbf{G} \times \mathbf{I}\right) \times \mathbf{G}^{-1} \times\left(\mathbf{B}_{\mathbf{z b}}-\mathbf{G} \times \mathbf{I}\right)\right] \text { be minimal. }
$$

\subsection{Estimation of the arc size and position}

We seek to compute (an approximation to) the true plate position from the reconstructed data. The problem can be expressed as follows: 
Given data values on an interval from $[0, L]$, find the step function that best approximates the data with the following constraints on the step function:

1) It is zero on $\left[0, Z_{a}\right]$ and on $\left[Z_{b}, L\right]$,

2) It has a constant value over $\left(Z_{a}, Z_{b}\right)$,

Here, $0 \leq \mathrm{Z}_{\mathrm{a}}<\mathrm{Z}_{\mathrm{b}} \leq \mathrm{L}$

This step function is then actually defined by only three numbers: $\mathrm{Z}_{\mathrm{a}}, \mathrm{Z}_{\mathrm{b}}$, and $\mathrm{i}^{*}$ (figure 4). This means that finding the best approximating step function is reduced to the problem of finding the three values, $Z_{a}, Z_{b}$, and $\mathrm{i}^{*}$ that define the best-fitting step function.

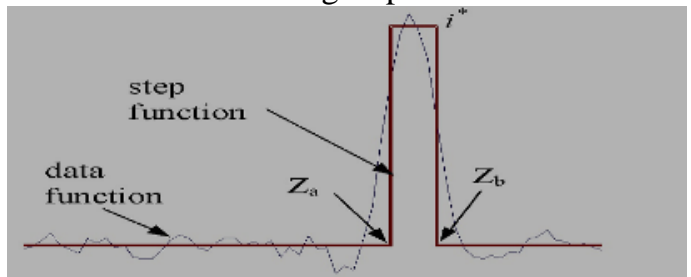

Fig. 4. Estimation of the plate position and width In this work, we defined the "best" approximation in a natural way, that is, as the best in a least squares sense. I.e., we minimize the sum of squares of the differences between the step function and the data. Actually, for any given values of $Z_{a}$ and $Z_{b}$, we can find $i^{*}$ immediately.

Since we are seeking the least squares solution, $\mathrm{i}^{*}$ must be the average value of the data over the interval. This is an elementary computation, and it is documented in [10], which shows that the least squares line (even a constant one like we have here) passes through the centroid of the data. So the least squares search is now reduced to a search over only two variables, $\mathrm{Z}_{\mathrm{a}}$ and $\mathrm{Z}_{\mathrm{b}}$.

\section{Experimental validation of the method}

Before studying the electric arc, we have decided to test the method by reconstructing the current density in stationary conducting plates. These plates are welded in a known position between two vertical electrodes. In the following we present three typical tests.

Test 1: using a wide conducting Plate: A copper plate with a width of $\ell=15 \mathrm{~mm}$ is welded to the two electrodes between the heights $Z_{a}=57 \mathrm{~mm}$ and $\mathrm{Z}_{\mathrm{b}}=72 \mathrm{~mm}$. The electrode spacing is equal to $\mathrm{L}=40 \mathrm{~mm}$ (figure 8). A current from the power source of maximum value $940 \mathrm{~A}$ flows through the plate. The wide plate simulates the diffuse mode of the arc.

Test 2: using a narrow conducting plate: This second test was carried out with a narrow copper plate with a width of $\ell=7.5 \mathrm{~mm}$, welded to the electrodes between the heights $Z_{a}=60 \mathrm{~mm}$ and $Z_{b}=67.5 \mathrm{~mm}$. It was traversed by a current of maximum value $\mathrm{I}=1015 \mathrm{~A}$. This allows studying the validity of the method for concentrated arc mode.

Test 3: using two conducting Plates: This test studies two current sheets, which are traversed by different value currents, and simulates the re-strike phenomena in a lowvoltage breaking device. We placed two plates in parallel, each one having a width of $\ell=8 \mathrm{~mm}$. The first one is made of stainless steel and is welded between the heights $Z_{\mathrm{a} 1}=24 \mathrm{~mm}$ and $\mathrm{Z}_{\mathrm{b} 1}=32 \mathrm{~mm}$, and the other one is made of copper and is welded between the heights $Z_{\mathrm{a} 2}=54 \mathrm{~mm}$ and $\mathrm{Z}_{\mathrm{b} 2}=62 \mathrm{~mm}$. The maximum value of the total current I is $1300 \mathrm{~A}$.

\section{Results}

\subsection{Reconstructed current densities}

Using the 64 effect Hall sensor, we have measured magnetic induction created by the conductor plates for the three tests, using the iterative deconvolution method we obtain the reconstructed current densities. Figures 5, 6 , and 7 , respectively, display the reconstructed current density and the theoretical current density for each test. It should be noted that the theoretical current density is assumed to have a uniform current distribution in the plate. Its value is equal to the ratio between the total current I, at a given time, and the plate width $\ell$.

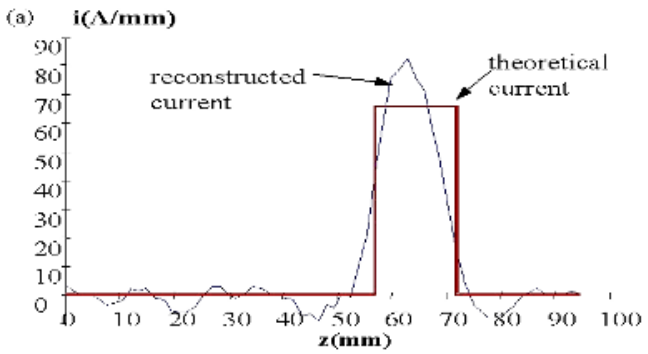

Fig.5. Results for the wide plate.

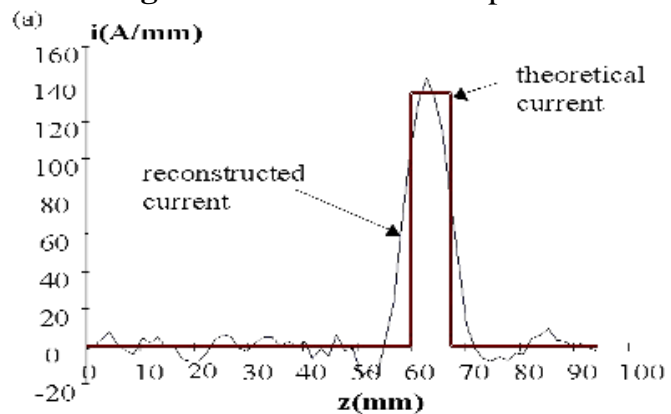

Fig. 6. Results for the narrow plate.

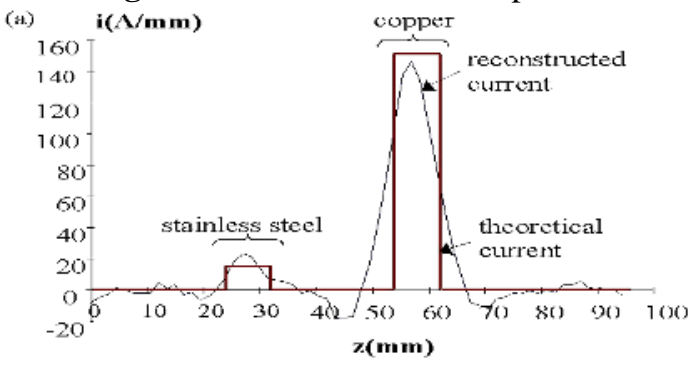

Fig.7. Results for the two current sheets.

\subsection{Plate position and size}

Using the estimation method presented in paragraph 2.4 and the reconstructed current densities, the size and the position of the plates, for each test are evaluated. Table 1 regroups these values. 


\subsection{Uncertainties analysis}

In order to discuss the result and to study the method precision, we evaluated, for each test, the relative uncertainties of the position and the size on the plate and value of the current density.

Precision on the value of the current density: The precision on the current density in the plate is calculated with the following relation:

$$
\Delta \mathrm{I}=\frac{\left|\mathrm{I}-\mathrm{I}_{\mathrm{rec}}\right|}{\mathrm{I}} \text {. }
$$

Table 1. Plates size and position resulting.

\begin{tabular}{|l|l|l|l|l|}
\hline \multicolumn{2}{|l|}{ Matrix method } & $\ell(\mathrm{mm})$ & $\mathrm{Z}_{\mathrm{a}}(\mathrm{mm})$ & $\mathrm{Z}_{\mathrm{b}}(\mathrm{mm})$ \\
\hline Wide plate & 13.68 & 56.27 & 69.95 \\
\hline \multicolumn{2}{|l|}{ Narrow plate } & 9.48 & 59.02 & 68.50 \\
\hline $\begin{array}{l}\text { Two } \\
\text { current } \\
\text { sheets }\end{array}$ & $\begin{array}{l}\text { Stainless } \\
\text { steel }\end{array}$ & 11.09 & 22.02 & 33.11 \\
\cline { 2 - 5 } & Copper & 10.74 & 53.45 & 64.19 \\
\hline
\end{tabular}

In this relation, I is the total current which flows through the circuit and $\mathrm{I}_{\mathrm{rec}}=\sum_{1}^{64} \mathrm{i}_{\mathrm{rec}}(\mathrm{k}) . \mathrm{i}_{\mathrm{rec}}(\mathrm{k})$ represents the reconstructed current density. The results are presented in Table 2.

Table 2. Precision on the current density value.

\begin{tabular}{|c|c|}
\cline { 2 - 2 } \multicolumn{1}{c|}{} & $\Delta \mathrm{I}(\%)$ \\
\hline Wide plate & 4.69 \\
\hline Narrow plate & 4.07 \\
\hline Two plates & 0.72 \\
\hline
\end{tabular}

Precision of the plate position and size: The estimated values of the plate positions and sizes are then compared to the true values of the position and the size of the plates. The differences between the estimated value and the true ones present the relative uncertainties $\left(\Delta \mathrm{Z}_{\mathrm{a}}, \Delta \mathrm{Z}_{\mathrm{b}}\right)$ on the plates position, and $(\Delta \ell)$ on the plate size. The results are presented in Table 3 .

Table 3. Precision on the plate size and position.

\begin{tabular}{|l|l|l|l|l|}
\cline { 2 - 4 } \multicolumn{1}{c|}{} & $\Delta \ell(\mathrm{mm})$ & $\Delta \mathrm{Z}_{\mathrm{a}}(\mathrm{mm})$ & $\Delta \mathrm{Z}_{\mathrm{b}}(\mathrm{mm})$ \\
\hline \multicolumn{2}{|l|}{ Wide plate } & 1.32 & 0.77 & 0.54 \\
\hline \multicolumn{2}{|c|}{ Narrow plate } & 1.98 & 0.52 & 2.50 \\
\hline \multirow{2}{*}{$\begin{array}{l}\text { Two } \\
\text { plates }\end{array}$} & $\begin{array}{l}\text { Stainless } \\
\text { steel }\end{array}$ & 3.09 & 1.98 & 1.12 \\
\cline { 2 - 5 } & Copper & 2.74 & 0.55 & 2.19 \\
\hline
\end{tabular}

\section{Discussion}

The reconstructed data show the position, the size and the current density values of the conductor with reasonable accuracy. However (figure 5, 6 and 7) the data fluctuate in space with a magnitude up to $10 \%$, resulting in identification non-zero current densities outside the region where electric current is actually flowing, this is probably result from measurement uncertainties. In practice there are two sources of measurement uncertainty can be inherent to the magnetic induction measurements and these are: the uncertainty in knowing the position where the magnetic induction is measured, and the uncertainty in the magnetic induction measurement itself. It was shown in [11] that these two errors affect strongly the uncertainty of the arc geometry and position reconstruction. Note that the method is valid under the assumption that the current density is constant through the plate along the direction of the electric current, and has a rectangular shape. Departures from these two ideal states may result in error in the result. The result can be improved by using others deconvolution method such as wavelet transform method.

\section{References}

1. V. Mestcheryakov, S. Akachev D. Podolosky, Structure of : arc in arc chute, (Proc.21th Int. Conf. on Electric Contacts, Zurich, 2002).

2. A. Sokolov, Contacts erosion of contacts dependent on arc discharge modes (Proc.21th Int. Conf. on Electric Contacts, Zurich, 2002).

3. C. Brdys, JP. Toumazet, A. Laurent, JL. Ponthenier, Optical and magnetic diagnostics of the electric arc dynamics in a low voltage circuit breaker Meas. Sci. Technol., 13, 1146-1153, (2002).

4. B. Bouvier, A deconvolution technique for B-dot Signals from a plasma-driven electromagnetic Launcher IEEE Trans plasma Sci, 17, 516-519, (1989).

5. N. Machkour, Magnetic method to characterize the current density in breaker arc, Fusion Science and Technology, 47, 784-789, (2005).

6. N. Machkour, Méthode de déconvolution appliquée à l'étude de la densité surfacique d'un arc électrique de coupure basse tension à partir de mesures magnétiques, (thesis of Blaise Pascal University, Clermont Ferrand, 2003).

7. JP. Toumazet, G. Vellaud, C. Brdys, S. Servant, A study of the influence of the walls nature on the behaviour of a low-voltage arc breaker by means of an inverse method. J Phys. D: Appl. Phys., 32, 121127, (1999).

8. H. Fenniri, A. Moineau, G. Delannay, The use of some iterative deconvolution algorithms to improve the spatial resolution of a flat magnetic sensor. Sensors and Actuators, 63, 7-13, (1997).

9. DG. Sutton, V. Kempi, Constrained least-square restoration and renogram deconvolution: a comparison by simulation Phys.Med.Biol., 37, 5367, (1992).

10. CM. Shakarji, Least Squares Fitting Algorithms of the NIST Algorithm Testing System, J. Res. Natl. Inst. Stand. Technol., 103, 633-641 (1998).

11. N. Machkour, G. Granet, G. Velleaud, N. Yashina, Simulation Studies of magnetic method applied to diagnose gliding arc, (Proceeding IEEE, 6th MSMW, Kharkov, 2007). 